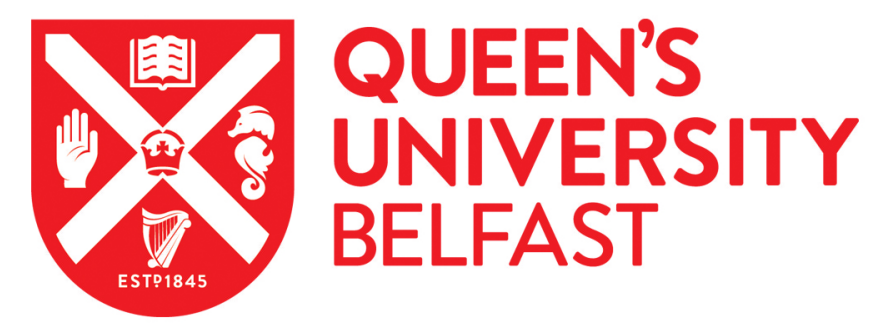

\title{
Simulation of distributed contact in string instruments: A modal expansion approach
}

van Walstijn, M., \& Bridges, J. (2016). Simulation of distributed contact in string instruments: A modal expansion approach. In 24th European Signal Processing Conference (Eusipco 2016) (pp. 1023-1027)

https://doi.org/10.1109/EUSIPCO.2016.7760403

Published in:

24th European Signal Processing Conference (Eusipco 2016)

Document Version:

Peer reviewed version

Queen's University Belfast - Research Portal:

Link to publication record in Queen's University Belfast Research Portal

Publisher rights

( $) 2016$ IEEE. Personal use of this material is permitted. Permission from IEEE must be obtained for all other uses, in any current or future media, including reprinting/republishing this material for advertising or promotional purposes, creating new collective works, for resale or redistribution to servers or lists, or reuse of any copyrighted component of this work in other works

\section{General rights}

Copyright for the publications made accessible via the Queen's University Belfast Research Portal is retained by the author(s) and / or other copyright owners and it is a condition of accessing these publications that users recognise and abide by the legal requirements associated with these rights.

Take down policy

The Research Portal is Queen's institutional repository that provides access to Queen's research output. Every effort has been made to ensure that content in the Research Portal does not infringe any person's rights, or applicable UK laws. If you discover content in the Research Portal that you believe breaches copyright or violates any law, please contact openaccess@qub.ac.uk. 


\title{
Simulation of Distributed Contact in String Instruments: a Modal Expansion Approach
}

\author{
(Invited Paper) \\ Maarten van Walstijn and Jamie Bridges \\ Sonic Arts Research Centre \\ School of Electronics, Electrical Engineering and Computer Science \\ Queen's University Belfast \\ Email: $\{m$.vanwalstijn, jbridges 05$\}$ @qub.ac.uk
}

\begin{abstract}
Impactive contact between a vibrating string and a barrier is a strongly nonlinear phenomenon that presents several challenges in the design of numerical models for simulation and sound synthesis of musical string instruments. These are addressed here by applying Hamiltonian methods to incorporate distributed contact forces into a modal framework for discretetime simulation of the dynamics of a stiff, damped string. The resulting algorithms have spectral accuracy, are unconditionally stable, and require solving a multivariate nonlinear equation that is guaranteed to have a unique solution. Exemplifying results are presented and discussed in terms of accuracy, convergence, and spurious high-frequency oscillations.
\end{abstract}

\section{INTRODUCTION}

Distributed contact between a vibrating string and a barrier is an intrinsic feature of many musical string instruments. The nature of the impactive interaction varies from sustained (e.g. string-bridge contact in Eastern instruments such as the sitar, tanpura, and the biwa) to more incidental (e.g. stringfingerboard contact in generating a 'slap bass' sound on the bass guitar). In all cases, collisions result in the generation of high-frequency components, which often are an important characteristic of the instrument.

Such contact is frequently simulated with digital waveguides, considering the collisions to be either inelastic [1]-[3] or semi-elastic [4], or even to assume a perfectly rigid barrier [5]. Methods based on modal expansion have also been proposed [6], [7]. Apart from [7], which studies impactive interaction at a single point of the string, relatively little attention is given in these studies to rigorous analysis of numerical stability. More recently, provably stable formulations have been derived in finite difference form, by construction of time-stepping schemes that respect the energy balance inherent to the underlying continuous-domain model (see, e.g. [8], [9]).

All of these models inevitably feature some form of discretisation error. For example, the tanpura models presented in [9], [10] exhibit severe mode detuning unless a high sampling frequency is used. Further numerical errors may arise due to the strongly nonlinear nature of the contact forces (simulated in energy-conserving formulations using a discrete gradient in either three-point [11] or two-point form [9], [10]), making numerical models prone to aliasing and spurious highfrequency oscillation.
To better address and further investigate these issues, this paper presents provably stable numerical formulations based on a modal approach, which allows eliminating mode detuning altogether and in addition facilitates exact modal damping. Section II outlines a continuous-domain description of the string model, specifying the contact forces in power-law form and modally expanding the string motion. Time discretisation is then performed in Section III by direct application of difference and sum operators to a first-order form of the mode differential equations, allowing direct control over useful energy properties, and yielding a two-point scheme. For comparison, a three-point form is also derived. The contact forces are incorporated using a discrete gradient at a finite set of barrier points. The two-point and three-point scheme are both shown to be strictly dissipative for non-zero damping constants. Spectral accuracy is achieved by adjusting the modal elasticity and damping constants in such a way that numerical dispersion and numerical attenuation are exactly compensated for. In Section IV, the accuracy of these formulations is tested against an analytical result and further investigated through simulation of the vibrations of a sitar-like stringbarrier configuration.

\section{Continuous-Domain Formulation}

\section{A. Model Equations}

The transverse motion for a stiff string, taking into account collisions with a barrier, may be described by:

$$
\rho A \frac{\partial^{2} y}{\partial t^{2}}=T \frac{\partial^{2} y}{\partial x^{2}}-E I \frac{\partial^{4} y}{\partial x^{4}}+\gamma(\beta) \frac{\partial y}{\partial t}+\mathcal{F}_{\mathrm{b}}(x, t),
$$

where $\rho, A, T, E$, and $I$ are the mass density, cross-sectional area, tension, Young's modulus, and moment of inertia, respectively. Following previous musical acoustics studies, contact is modelled with a power law. Here the force density

$$
\mathcal{F}_{\mathrm{b}}(x, t)=-k_{\mathrm{b}}\left\lfloor\left\{y_{\mathrm{b}}(x)-y(x, t)\right\}^{\chi}\right\rfloor
$$

models string-barrier collisions over the spatial domain of the barrier profile $y_{\mathrm{b}}(x)$, where $k_{\mathrm{b}}$ and $\chi$ are the contact parameters. The term $\left\lfloor y^{\chi}\right\rfloor$ denotes $u(y) \cdot y^{\chi}$, where $u(y)$ is the unit step function. In preparation of discretisation, we write the contact force density in Hamiltonian form

$$
\mathcal{F}_{\mathrm{b}}(x, t)=-\frac{\partial \mathcal{V}_{\mathrm{b}}}{\partial y}
$$


where

$$
\mathcal{V}_{\mathrm{b}}(y)=\frac{k_{\mathrm{b}}}{\chi+1}\left\lfloor\left\{y_{\mathrm{b}}(x)-y(x, t)\right\}^{\chi+1}\right\rfloor
$$

is the contact potential energy density. Frequency-dependent string damping is incorporated by defining parameter $\gamma$ as a function of the wave number $\beta$

$$
\gamma(\beta)=2 \rho A\left[\sigma_{0}+\left(\sigma_{1}+\sigma_{3} \beta^{2}\right)|\beta|\right],
$$

where the fit parameters $\sigma_{0,1,3}$ can be directly related to the three-parameter damping formulation by Woodhouse in [12].

\section{B. Modal Expansion}

The solution to (1) can be expressed as a superposition of modal displacements:

$$
y(x, t)=\sum_{i=1}^{M} v_{i}(x) \bar{y}_{i}(t),
$$

where $\bar{y}_{i}(t)$ denotes the displacement of the $i$ th mode, $v_{i}(x)=$ $\sin (i \pi x / L)$ is the corresponding modal shape (spatial eigenfunction) for simply supported boundary conditions, and $M$ is the number of modes taken into account (for an exact solution, $M \rightarrow \infty)$. After substitution of (6) into (1) and applying a spatial integral over the length of the string, one obtains that the dynamics of each of the modes is governed by

$$
\bar{m} \frac{\partial^{2} \bar{y}_{i}}{\partial t^{2}}+\bar{r}_{i} \frac{\partial \bar{y}_{i}}{\partial t}+\bar{k}_{i} \bar{y}_{i}(t)=\bar{F}_{\mathrm{b}, i}(t),
$$

in which $\bar{m}=(\rho A L) / 2$ is the modal mass (which is the same for all modes), and where $\bar{k}_{i}=\frac{1}{2} L\left(E I \beta_{i}^{4}+T \beta_{i}^{2}\right)$ and $\bar{r}_{i}=$ $\frac{1}{2} L \gamma\left(\beta_{i}\right)$ are the elastic and damping constants of the mode, respectively. Within the constraint $\bar{r}_{i}<2 \sqrt{\bar{k}_{i} \bar{m}}$ the modal frequencies $\left(\omega_{i}\right)$ and decay rates $\left(\alpha_{i}\right)$ are

$$
\omega_{i}=\sqrt{\bar{k}_{i} / \bar{m}_{i}-\alpha_{i}^{2}}, \quad \alpha_{i}=\frac{\bar{r}_{i}}{2 \bar{m}}=\sigma_{0}+\sigma_{1} \beta_{i}+\sigma_{3} \beta_{i}^{3} .
$$

The modal driving forces in (7) are

$$
\bar{F}_{\mathrm{b}, i}(t)=\int_{0}^{L} v_{i}(x) \mathcal{F}_{\mathrm{b}}(x, t) d x .
$$

\section{NUMERICAL FORMULATION}

\section{A. Discretisation in Space}

Given that $\mathcal{F}_{\mathrm{b}}(x, t)$ is non-analytic, we approximate the integral in (9) as a Riemann sum:

$$
\bar{F}_{\mathrm{b}, i}(t) \approx \sum_{k=1}^{K} v_{i, k} \mathcal{F}_{\mathrm{b}, k}(t) \Delta_{\mathrm{x}}
$$

where $\mathcal{F}_{\mathrm{b}, k}(t) \equiv \mathcal{F}_{\mathrm{b}}\left(x_{k}, t\right)$ and $v_{i, k} \equiv v_{i}\left(x_{k}\right)$, with $K$ chosen contact points $x_{k}$ spaced $\Delta_{x}$ apart along the $x$-axis.

\section{B. Discretisation in Time}

In order to facilitate the derivation of a two-point scheme, equation (7) is first re-formulated in first-order form:

$$
\begin{aligned}
\frac{\partial \bar{y}_{i}}{\partial t} & =\frac{\bar{p}_{i}(t)}{\bar{m}}, \\
\frac{\partial \bar{p}_{i}}{\partial t} & =-\bar{k}_{i} \bar{y}_{i}(t)-\bar{r}_{i} \frac{\partial \bar{y}_{i}}{\partial t}+\Delta_{\mathrm{x}} \sum_{k=1}^{K} v_{i, k} \mathcal{F}_{\mathrm{b}, k}(t),
\end{aligned}
$$

in which $\bar{p}_{i}(t)$ represents the momentum. Taking the temporal step as $\Delta_{\mathrm{t}}=f_{\mathrm{s}}^{-1}$, where $f_{\mathrm{s}}$ is the sampling frequency, the displacement at discrete time instances indexed with $n$ is denoted $y^{n} \equiv y\left(n \Delta_{\mathrm{t}}\right)$. Using the difference and sum operators

$$
\begin{aligned}
& \delta y^{n}=y^{n+\frac{1}{2}}-\left.y^{n-\frac{1}{2}} \approx \Delta_{\mathrm{t}} \frac{\partial y}{\partial t}\right|_{t=n \Delta_{\mathrm{t}}}, \\
& \mu y^{n}=y^{n+\frac{1}{2}}+\left.y^{n-\frac{1}{2}} \approx 2 y\right|_{t=n \Delta_{\mathrm{t}}},
\end{aligned}
$$

equations $(11,12)$ can be discretised as

$$
\begin{aligned}
& \frac{\delta \bar{y}_{i}^{n+\frac{1}{2}}}{\Delta_{\mathrm{t}}}=\frac{\mu \bar{p}_{i}^{n+\frac{1}{2}}}{2 \bar{m}}, \\
& \frac{\delta \bar{p}_{i}^{n+\frac{1}{2}}}{\Delta_{\mathrm{t}}}=-\bar{k}_{i} \frac{\mu \bar{y}_{i}^{n+\frac{1}{2}}}{2}-\bar{r}_{i} \frac{\delta \bar{y}_{i}^{n+\frac{1}{2}}}{\Delta_{\mathrm{t}}}+\Delta_{\mathrm{x}} \sum_{k=1}^{K} v_{i, k} \mathcal{F}_{\mathrm{b}, k}^{n+\frac{1}{2}},
\end{aligned}
$$

which is equivalent to applying the trapezoidal rule. The nonanalytic form of the contact force warrants special treatment regarding stability [8], [9]. A suitable term is obtained by discretising (3), yielding the two-point discrete gradient:

$$
\mathcal{F}_{\mathrm{b}, k}^{n+\frac{1}{2}}=-\frac{\delta \mathcal{V}_{\mathrm{b}, k}^{n+\frac{1}{2}}}{\delta y_{\mathrm{b}, k}^{n+\frac{1}{2}}}=-\frac{\mathcal{V}_{\mathrm{b}, k}\left(y_{\mathrm{b}}^{n+1}\right)-\mathcal{V}_{\mathrm{b}}\left(y_{\mathrm{b}, k}^{n}\right)}{y_{\mathrm{b}, k}^{n+1}-y_{\mathrm{b}, k}^{n}} .
$$

Note that in the limit $y_{\mathrm{b}, k}^{n+1} \rightarrow y_{\mathrm{b}, k}^{n}$, (17) must be evaluated as $-\mathcal{V}_{\mathrm{b}}^{\prime}\left(y_{\mathrm{b}, k}^{n}\right)$, where $\mathcal{V}_{\mathrm{b}}^{\prime}(y) \equiv \partial \mathcal{V}_{\mathrm{b}} / \partial y$. Using the scaled momentum value $\bar{q}_{i}^{n}=\left(\Delta_{\mathrm{t}} /(2 \bar{m})\right) \bar{p}_{i}^{n}$, the system equations $(15,16)$ can be written more conveniently as

$$
\begin{aligned}
& \delta \bar{y}_{i}^{n+\frac{1}{2}}=\mu \bar{q}_{i}^{n+\frac{1}{2}}, \\
& \delta \bar{q}_{i}^{n+\frac{1}{2}}=-a_{i} \mu \bar{y}_{i}^{n+\frac{1}{2}}-b_{i} \delta \bar{y}_{i}^{n+\frac{1}{2}}+\xi \Delta_{\mathrm{x}} \sum_{k=1}^{K} v_{i, k} \mathcal{F}_{\mathrm{b}, k}^{n+\frac{1}{2}},
\end{aligned}
$$

where $\xi=\Delta_{\mathrm{t}}^{2} /(2 m), a_{i}=\bar{k}_{i} \Delta_{\mathrm{t}}^{2} /(4 \bar{m})$, and $b_{i}=\bar{r}_{i} \Delta_{\mathrm{t}} /(2 \bar{m})$. Once the contact force densities $\mathcal{F}_{\mathrm{b}, k}^{n+\frac{1}{2}}$ are known, the dynamics of each mode can be simulated by solving for $\bar{y}_{i}^{n+1}$ and $\bar{q}_{i}^{n+1}$ at each time step. To eliminate numerical dispersion, a 'pre-warping' procedure is first carried out for each mode, by replacing the elasticity and damping values as follows:

$$
\bar{k}_{i} \rightarrow \bar{k}_{i}^{*}=\frac{4 \bar{m} a_{i}^{*}}{\Delta_{\mathrm{t}}^{2}}, \quad \bar{r}_{i} \rightarrow \bar{r}_{i}^{*}=\frac{2 \bar{m} b_{i}^{*}}{\Delta_{\mathrm{t}}},
$$

where the adjusted coefficients that replace $\left(a_{i}, b_{i}\right)$ in (19) are

$$
a_{i}^{*}=\frac{1-2 R_{i} \Omega_{i}+R_{i}^{2}}{1+2 R_{i} \Omega_{i}+R_{i}^{2}}, \quad b_{i}^{*}=\frac{2\left(1-R_{i}^{2}\right)}{1+2 R_{i} \Omega_{i}+R_{i}^{2}},
$$

with $R_{i}=\exp \left(-\alpha_{i} \Delta_{\mathrm{t}}\right)$ and $\Omega_{i}=\cos \left(\omega_{i} \Delta_{\mathrm{t}}\right)$. Using frequencydomain analysis it can be shown that this ensures that the modal parameters $\left(\omega_{i}, \alpha_{i}\right)$ are exactly preserved under discretisation [13]. Note that the bandwidth constraint $\omega_{i}<\pi / \Delta_{t}$ must be respected, which sets an upper limit to $M$ in the modal series expansion in (6).

\section{A Vector-Matrix Update Form}

Consider now an $M \times K$ matrix $\mathbf{V}$ with rows that hold the (spatially sampled) modal shapes for $K$ points along the $x$-axis. From (6) and (10) it then follows that

$$
\mathbf{y}^{n}=\mathbf{V} \overline{\mathbf{y}}^{n}, \quad \overline{\mathbf{F}}_{\mathrm{b}}^{n+\frac{1}{2}}=\Delta_{\mathrm{x}} \mathbf{V}^{\top} \mathcal{F}_{\mathrm{b}}^{n+\frac{1}{2}},
$$


where $\mathbf{y}^{n}$ is a column vector holding the string displacements at the $K$ points, and $\overline{\mathbf{y}}^{n}$ is a column vector holding the $M$ modal displacements; similarly $\overline{\mathbf{F}}_{\mathrm{b}}^{n+\frac{1}{2}}$ and $\overline{\mathcal{F}}_{\mathrm{b}}^{n+\frac{1}{2}}$ are column vectors holding $M$ modal driving forces and $K$ contact force densities, respectively. A vector-matrix form of $(18,19)$ can then be written as

$$
\begin{aligned}
& \delta \overline{\mathbf{y}}^{n+\frac{1}{2}}=\mu \overline{\mathbf{q}}^{n+\frac{1}{2}}, \\
& \delta \overline{\mathbf{q}}^{n+\frac{1}{2}}=-\mathbf{A} \mu \overline{\mathbf{y}}^{n+\frac{1}{2}}-\mathbf{B} \delta \overline{\mathbf{y}}^{n+\frac{1}{2}}+\xi \Delta_{\mathrm{x}} \mathbf{V}^{\top} \mathcal{F}_{\mathrm{b}}^{n+\frac{1}{2}},
\end{aligned}
$$

where $\mathbf{A}$ and $\mathbf{B}$ are diagonal matrices with entries $A_{i i}=a_{i}^{*}$ and $B_{i i}=b_{i}^{*}$. A convenient vector-matrix update is found by using (23) to define $\overline{\mathbf{s}}=\delta \overline{\mathbf{y}}^{n+\frac{1}{2}}=\mu \overline{\mathbf{q}}^{n+\frac{1}{2}}$, and substituting

$$
\overline{\mathbf{y}}^{n+1}=\overline{\mathbf{s}}+\overline{\mathbf{y}}^{n}, \quad \overline{\mathbf{q}}^{n+1}=\overline{\mathbf{s}}-\overline{\mathbf{q}}^{n},
$$

in (24), which allows solving for $\overline{\mathrm{s}}$ with

$$
\overline{\mathbf{s}}=\overline{\mathbf{u}}+\xi \Delta_{\mathrm{x}} \mathbf{C V}^{\top} \mathcal{F}_{\mathrm{b}}^{n+\frac{1}{2}},
$$

with $\mathbf{C}=(\mathbf{I}+\mathbf{A}+\mathbf{B})^{-1}$, and where $\overline{\mathbf{u}}=\mathbf{C}\left[\left(\overline{\mathbf{q}}^{n}-\mathbf{A} \overline{\mathbf{y}^{n}}\right)\right]$. Hence once the contact force density vector $\mathcal{F}_{\mathrm{b}}^{n+\frac{1}{2}}$ is known, eq. (26) immediately yields the step vector $\overline{\mathbf{s}}$, after which the modal state vectors $\overline{\mathbf{y}}^{n+1}$ and $\overline{\mathbf{q}}^{n+1}$ can be updated using (25). Note that while $\mathbf{A}, \mathbf{B}$, and $\mathbf{C}$ are diagonal, $\mathbf{V}$ is a full matrix.

\section{Solving for the Contact Force Densities}

Pre-multiplying (26) by $\mathbf{V}$ yields

$$
\mathbf{s}=\mathbf{u}+\xi \Delta_{\mathrm{x}} \mathbf{W} \mathcal{F}_{\mathrm{b}}^{n+\frac{1}{2}}
$$

where $\mathbf{s}=\mathbf{V} \overline{\mathbf{s}}, \mathbf{u}=\mathbf{V} \overline{\mathbf{u}}$, and $\mathbf{W}=\mathbf{V C V}^{\top}$. After substituting (17) one obtains the multivariate nonlinear equation

$$
\mathbf{s}-\mathbf{u}+\Delta_{\mathrm{x}} \xi \mathbf{W} \frac{\mathcal{V}_{\mathrm{b}}\left(\mathbf{s}+\mathbf{y}^{n}\right)-\mathcal{V}_{\mathrm{b}}\left(\mathbf{y}^{n}\right)}{\mathbf{s}}=\mathbf{0} .
$$

This can be solved iteratively with Newton's method, employing the Jabobian $\mathbf{J}=\mathbf{I}+\mathbf{W D}$, where

$$
\mathbf{D}=\Delta_{\mathrm{x}} \xi \frac{\mathbf{s} \circ \mathcal{V}_{\mathrm{b}}^{\prime}\left(\mathbf{s}+\mathbf{y}^{n}\right)-\mathcal{V}_{\mathrm{b}}\left(\mathbf{s}+\mathbf{y}^{n}\right)+\mathcal{V}_{\mathrm{b}}\left(\mathbf{y}^{n}\right)}{\mathbf{s} \circ \mathbf{s}},
$$

in which the division is componentwise and where $\circ$ denotes componentwise multiplication. Once $\mathbf{s}$ is known, the corresponding force density vector $\mathcal{F}_{\mathrm{b}}^{n+\frac{1}{2}}$ can be plugged into (26), allowing an update of $\overline{\mathbf{y}}^{n+1}, \overline{\mathbf{q}}^{n+1}$ and $\mathbf{y}^{n+1}$.

\section{E. Numerical Energy and Stability}

The energy (Hamiltonian) of mode $i$ at $t=n \Delta_{\mathrm{t}}$ is

$$
\bar{H}_{i}^{n}=\frac{1}{2} \bar{m}^{-1}\left(\bar{p}_{i}^{n}\right)^{2}+\frac{1}{2} \bar{k}_{i}^{*}\left(\bar{y}_{i}^{n}\right)^{2} .
$$

This is a quadratic form in $\bar{p}_{i}^{n}$ and $\bar{y}_{i}^{n}$ that is non-negative since $a_{i}^{*} \geq 0$ therefore $\bar{k}_{i}^{*} \geq 0$. The total numerical energy at time $t=n \Delta_{\mathrm{t}}$ can be formulated as the sum of all the modal energies plus the spatial integral over the contact potentials:

$$
\begin{aligned}
H^{n} & =\sum_{i=1}^{M} \bar{H}_{i}^{n}+\sum_{k=1}^{K} \mathcal{V}_{\mathrm{b}}\left(y^{n}\right) \Delta_{\mathrm{x}} \\
& =\xi^{-1}\left[\left(\overline{\mathbf{q}}^{n}\right)^{\top} \overline{\mathbf{q}}^{n}+\left(\overline{\mathbf{y}}^{n}\right)^{\top} \mathbf{A} \overline{\mathbf{y}}^{n}\right]+\Delta_{\mathrm{x}} \mathbf{1}^{\top} \mathcal{V}_{\mathrm{b}}\left(\mathbf{y}^{n}\right),
\end{aligned}
$$

where 1 denotes a vector with all $K$ elements equal to 1 . To arrive at a global energy balance, pre-multiply the left-hand side of equation (24) by $\mu \overline{\mathbf{q}}^{n+\frac{1}{2}}$ and the right-hand side by $\delta \overline{\mathbf{y}}^{n+\frac{1}{2}}$, yielding

$$
\Delta_{\mathrm{t}}^{-1}\left[H^{n+1}-H^{n}\right]=-Q^{n+\frac{1}{2}},
$$

where

$$
\left.Q^{n+\frac{1}{2}}=\left(\xi \Delta_{\mathrm{t}}\right)^{-1}\left[\overline{\mathbf{y}}^{n+\frac{1}{2}}\right)^{\top} \mathbf{B} \delta \overline{\mathbf{y}}^{n+\frac{1}{2}}\right]
$$

is the power dissipated across the time step. Under the damping constraint introduced in Section II-B, the diagonal matrices $\mathbf{A}$ and $\mathbf{B}$ are real-valued and positive definite, in which case both $H^{n}$ and $Q^{n+\frac{1}{2}}$ are non-negative. Hence the system can only be dissipative, or - in the absence of damping - conservative, which directly implies numerical stability.

\section{F. Existence, Uniqueness and Convergence}

It is generally not a given that a multivariate nonlinear equation arising in an implicit scheme actually has a solution, and that if it does exist that it is unique [14]. The condition for a unique solution is that the Jacobian is positive definite [15], which holds for $\mathbf{J}$ as can be shown as follows. For any real non-zero column vector $\mathbf{z}$ of length $K$ we have

$$
\mathbf{z}^{\top} \mathbf{W} \mathbf{z}=\left(\mathbf{V}^{\top} \mathbf{z}\right)^{\top} \mathbf{C}\left(\mathbf{V}^{\top} \mathbf{z}\right)>0,
$$

because $\mathbf{C}$ is diagonal with positive elements. The term WD is thus the product of a positive definite matrix $(\mathbf{W})$ and a diagonal positive semi-definite matrix $(\mathbf{D})$; such a product is positive semi-definite under the condition that it is normal [16], which is satisfied here since WD is a real square symmetric matrix. It follows that $\mathbf{z}^{\top} \mathbf{J} \mathbf{z}=\mathbf{z}^{\top} \mathbf{z}+\mathbf{z}^{\top} \mathbf{W D z}>0$. However $\mathbf{J}$ is not generally an M-matrix, thus the condition for global convergence of Newton's method is not met [17]. In other words, in order to converge, the initial iterate must lie sufficiently close to the actual solution. Quantifying this condition for specific cases is notoriously difficult, but the simulations indicate that using the previous value of $\mathbf{s}$ as the initial iterate has proven fairly robust when $\Delta_{\mathrm{t}} \leq 1 / 44.1 \mathrm{~ms}$.

\section{G. Alternative Discretisation: A Three-Point Scheme}

For the purpose of comparison, it is worthwhile considering an alternative approach that starts by directly discretising (7). For order-preserving methods, this will generally lead to a socalled three-point scheme, which utilises both $\overline{\mathbf{y}}^{n}$ and $\overline{\mathbf{y}}^{n-1}$ in the update of the new value $\overline{\mathbf{y}}^{n+1}$. Here we discretise (7) as follows:

$$
\bar{m} \frac{\delta \delta \bar{y}_{i}^{n}}{\Delta_{\mathrm{t}}^{2}}+\bar{r}_{i} \frac{\mu \delta \bar{y}_{i}^{n}}{2 \Delta_{\mathrm{t}}}+\bar{k}_{i} \bar{y}_{i}^{n}=\bar{F}_{i}^{n} .
$$

To counter-act the numerical dispersion for this scheme, the following adjusted modal elasticity and damping values have to be used

$$
\bar{k}_{i}^{*}=\frac{2 \bar{m}}{\Delta_{\mathrm{t}}^{2}}\left[1-\frac{2 R_{i} \cos \left(\omega_{i} \Delta_{\mathrm{t}}\right)}{1+R_{i}^{2}}\right], \bar{r}_{i}^{*}=\frac{2 \bar{m}}{\Delta_{\mathrm{t}}}\left[\frac{1-R_{i}^{2}}{1+R_{i}^{2}}\right] .
$$

The contact force densities now have to be formulated at time $t=n \Delta_{\mathrm{t}}$. The natural choice for this is

$$
\mathcal{F}_{\mathrm{b}, k}^{n}=-\frac{\mu \delta \mathcal{V}_{\mathrm{b}, k}^{n}}{\mu \delta y_{\mathrm{b}, k}^{n}}=-\frac{\mathcal{V}_{\mathrm{b}}\left(y_{\mathrm{b}, k}^{n+1}\right)-\mathcal{V}_{\mathrm{b}, k}\left(y_{\mathrm{b}, k}^{n-1}\right)}{y_{\mathrm{b}, k}^{n+1}-y_{\mathrm{b}, k}^{n-1}} .
$$



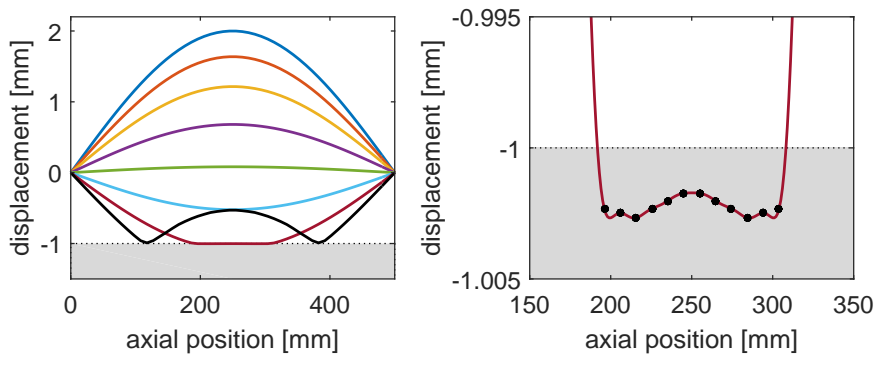

Fig. 1. Snapshots of the string motion of an ideal string colliding with a straight barrier, with contact parameters $k_{\mathrm{b}}=10^{9} \mathrm{~N} / \mathrm{m}^{2}$ and $\chi=1$ and string parameters $L=0.5 \mathrm{~m}, \rho A=5 \times 10^{-4} \mathrm{~kg} / \mathrm{m}$, and $T=64 \mathrm{~N}$. Left: full string profile. The snapshots were taken at 0ms (dark blue), $0.272 \mathrm{~ms}$ (red), $0.408 \mathrm{~ms}$ (yellow), 0.544ms (purple), 0.680ms (green), 0.816ms (light blue), 0.952ms (dark red), and 1.088ms (black). Right: zoomed view of the contact zone at $t=0.952 \mathrm{~ms}$; the black dots indicate the discrete spatial points of the model. In both plots, the grey shaded area represents the barrier.

As before, these equations can be combined in vector-matrix form. Now taking $\overline{\mathbf{s}}=\overline{\mathbf{y}}^{n+1}-\overline{\mathbf{y}}^{n-1}$, one arrives at

$$
\overline{\mathbf{s}}^{n+1}=\overline{\mathbf{u}}+\xi \Delta_{\mathrm{x}} \mathbf{C} \mathbf{V}^{\top} \mathcal{F}^{n},
$$

where $\overline{\mathbf{u}}=\mathbf{A} \overline{\mathbf{y}}^{n}-\mathbf{C} \overline{\mathbf{y}}^{n-1}$, with $A_{i i}=2 R_{i} \cos \left(\omega_{i} \Delta_{\mathrm{t}}\right)$ and $C_{i i}=1+R_{i}^{2}$. The nonlinear equation to be solved is

$$
\mathbf{s}-\mathbf{u}+\Delta_{\mathrm{x}} \xi \mathbf{W} \frac{\mathcal{V}_{\mathrm{b}}\left(\mathbf{s}+\mathbf{y}^{n-1}\right)-\mathcal{V}_{\mathrm{b}}\left(\mathbf{y}^{n-1}\right)}{\mathbf{s}}=\mathbf{0}
$$

with $\mathbf{u}=\mathbf{V} \overline{\mathbf{u}}$ and where $\mathbf{W}=\mathbf{V C V}^{\top}$ is positive semidefinite, thus (39) has a unique solution. A numerical energy balance again exists, however this time with an energy-like quantity defined at mid-point, i.e.

$$
\Delta_{\mathrm{t}}^{-1}\left[H^{n+\frac{1}{2}}-H^{n-\frac{1}{2}}\right]=-Q^{n}
$$

where

$$
\begin{aligned}
H^{n+\frac{1}{2}}= & \frac{1}{4} \xi^{-1}\left[\left(\delta \overline{\mathbf{y}}^{n+\frac{1}{2}}\right)^{\top} \delta \overline{\mathbf{y}}^{n+\frac{1}{2}}+\left(\overline{\mathbf{y}}^{n+1}\right)^{\top} \Upsilon \boldsymbol{\Upsilon}_{\overline{\mathbf{y}}}^{n}\right] \\
& +\frac{1}{2} \Delta_{\mathrm{x}} \mathbf{1}^{\top}\left[\mathcal{V}_{\mathrm{b}}\left(\mathbf{y}^{n+1}\right)+\mathcal{V}_{\mathrm{b}}\left(\mathbf{y}^{n}\right)\right], \\
Q^{n}= & \frac{1}{4} \xi^{-1} \Delta_{\mathrm{t}}{ }^{-1}\left(\delta \delta \overline{\mathbf{y}}^{n}\right)^{\top} \boldsymbol{\Lambda} \delta \delta \overline{\mathbf{y}}^{n} .
\end{aligned}
$$

The diagonal matrices $\Upsilon$ and $\boldsymbol{\Gamma}$ in $(41,42)$ have the elements $\Upsilon_{i i}=\bar{k}_{i}^{*} \Delta_{\mathrm{t}}^{2} / \bar{m}$ and $\Lambda_{i i}=\bar{r}_{i}^{*} \Delta_{\mathrm{t}} /(2 \bar{m})$, respectively. Even though the string potential energy term in (41) can become negative, the numerical Hamiltonian $H^{n+\frac{1}{2}}$ is guaranteed nonnegative due to the quadratic form of the modal energy terms in combination with the fact that $\left|\bar{k}_{i}^{*} \Delta_{\mathrm{t}}^{2} / \bar{m}-2\right| \leq 2$.

\section{Simulation Results}

\section{A. An Ideal String Colliding with a Straight Barrier}

In order to validate the numerical models and test their general behaviours, they are applied to the case of an ideal string (i.e. no stiffness or damping) colliding with a straight rigid barrier. It is known that if the string is initialised with its first mode shape, with the amplitude twice as large as the distance between the string and the barrier, then a periodic motion results, with the period 1.5 times longer than that of an unimpeded string [18]. Fig. 1 shows a selection of snapshots during the first $1.1 \mathrm{~ms}$ of the simulation using the two-point scheme with a $44.1 \mathrm{kHz}$ sampling frequency and
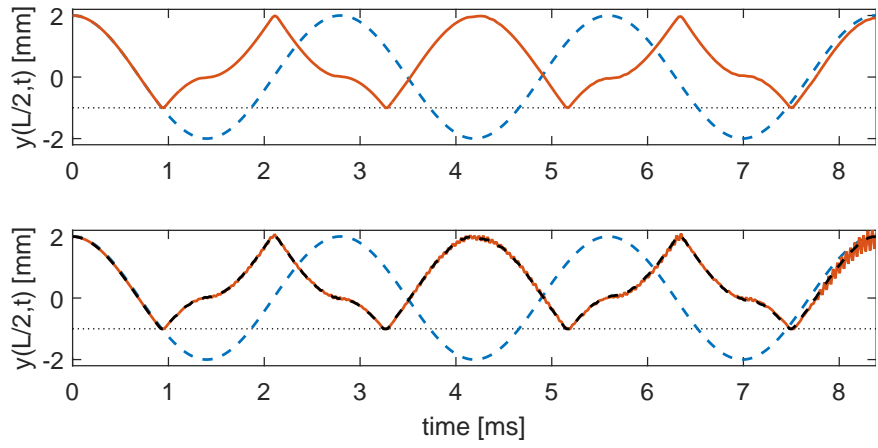

Fig. 2. String displacement at $x=L / 2$, as simulated with $f_{\mathrm{s}}=44.1 \mathrm{kHz}$ (red line). The blue dashed line indicates the displacement for free string motion. Top: two-point scheme. Bottom: three-point scheme; the black dashed line indicates the result obtained with the mode series truncated at $20 \mathrm{kHz}$.

setting $K=M$. As can be seen from the right plot, the string compression is of the order of micrometres in this case.

Fig. 2 plots the two-point and three-point scheme against the analytical result for an unimpeded string. For a rigid boundary, the curves should match up after two periods of oscillation. The two-point scheme produces the correct waveform but with a slightly longer cycle time. This is due to the boundary not being perfectly rigid, resulting in slightly elongated collision times than would have been the case for $k_{\mathrm{b}} \rightarrow \infty$. The threepoint scheme gives a similar result, but with spurious highfrequency oscillations appearing with increasing amplitude during the simulation. This can be remedied in this case by reducing the number of modes, for example by truncating the mode series at $20 \mathrm{kHz}$ (see the black dashed in the lower plot of 2). It is noted however that in other cases the mode cut-off has to be as low as $f_{\mathrm{s}} / 4$ in order to avoid such artefacts.

\section{B. A Sitar-like String-Barrier Configuration}

A Sitar string experiences sustained impactive interaction with a curved bridge [19]. Such a configuration is modelled here, be it with altered dimensions for clarity/visibility. Using again a unity exponent in the contact law, the value for $k_{\mathrm{b}}$ is approximately of the order of the smallest of the Young's moduli of the materials involved, and as such set here to $10^{9} \mathrm{~N} / \mathrm{m}^{2}$. Fig. 3 shows the string motion during the first $1.3 \mathrm{~ms}$, as simulated using the two-point scheme at $44.1 \mathrm{kHz}$. For both schemes, the modes were truncated at $20 \mathrm{kHz}$.

The evolution of the string force at $x=L$, which can be considered as a first approximation to the sound of the instrument, is shown in Fig. 4. As the comparisons with heavily oversampled results indicate, the two-point scheme simulation again exhibits less spurious oscillation.

\section{CONCLUDING REMARKS}

Distributed string-barrier contact has been incorporated in a provably stable manner in a numerical model based on modal expansion of the solution to the equation of motion of a stiff, lossy string. Of the two presented ways of discretising the equations, the two-point scheme employs the more accurate 

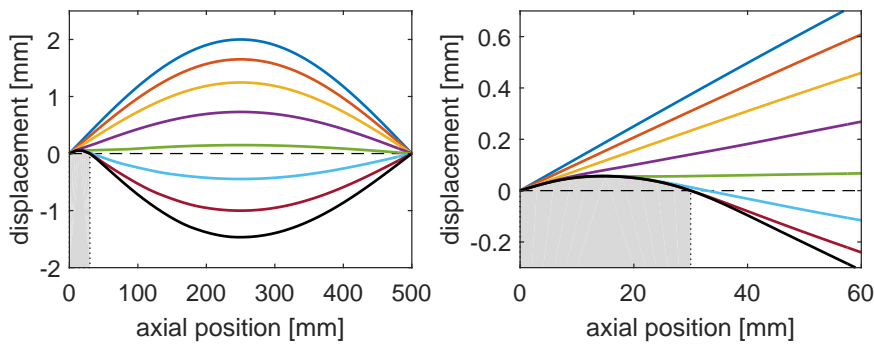

Fig. 3. Snapshots of the string motion of a stiff, damped string colliding with a $30 \mathrm{~mm}$ long parabolic barrier with profile $y_{\mathrm{b}}(x)=-\frac{1}{4} x(x-0.03)$, using $K=10$. The contact and string parameters are $k_{\mathrm{b}}=10^{9} \mathrm{~N} / \mathrm{m}^{2}, \chi=1$, $L=0.5 \mathrm{~m}, \rho A=5.55 \times 10^{-4} \mathrm{~kg} / \mathrm{m}, E I=7.95 \times 10^{5} \mathrm{Nm}^{2}, T=50 \mathrm{~N}$, $\sigma_{0}=0.6 \mathrm{~s}^{-1}, \sigma_{1}=6.5 \times 10^{-3} \mathrm{~m} / \mathrm{s}$, and $\sigma_{3}=5.0 \times 10^{-6} \mathrm{~m}^{3} / \mathrm{s}$. Left: full string profile. Right: zoomed view of the contact zone. The black dashed line in both plots indicates the string equilibrium. The snapshots were taken at Oms (dark blue), 0.317ms (red), 0.476ms (yellow), 0.635ms (purple), 0.793ms (green), 0.952ms (light blue), 1.111ms (dark red), and 1.270ms (black).

approximation of the contact forces. The simulation results indicate that this brings benefits regarding avoidance of spurious high-frequency oscillations. A Matlab code has been made available on the accompanying webpage ${ }^{1}$ allowing readers to explore these findings in more detail.

For simplicity, contact damping was omitted here but adding this (using a Hunt-Crossely form as in [10]) in order to model semi-elastic collisions is straightforward. The maximum number of iterations required for solving the nonlinear equation to a machine-precision tolerance in $\mathbf{s}$ is typically about 9 for the two-point scheme and 12 for the three-point scheme. In practice such extreme precision may not be necessary and in many cases the maximum number of iterations can be set to a slightly lower value. An important additional note to make here is that both schemes may suffer from non-convergence of the iterative solver; for example, this occurs with the sitar-like configuration for $f_{\mathrm{s}}=44.1 \mathrm{kHz}$ when the contact elasticity is increased to $k_{\mathrm{b}}=10^{10} \mathrm{~N} / \mathrm{m}^{2}$ or higher. In such cases, a damped version of Newton's method [17] can be employed, at the cost of a slight increase in computational effort.

In comparison with finite-difference schemes, the proposed models achieve a higher accuracy in the modal parameters. However this has to be weighed up against the advantage of finite difference models systematically having sparse system matrices. In particular the fact that the Jacobian of the iterative solver used in the modal formulations presented here is generally non-sparse implies computational load ramifications.

Regarding model extensions, of particular interest are (a) adding a tension modulation term, bringing with it a range of further nonlinear effects [14], and (b) also modelling a stopping finger, and for some instruments, a set of frets. As recently discussed in a finite difference context by Bilbao and Torin [20], such a more general setting presents increased challenges regarding solving the multivariate non-linear equation (i.e. issues related to uniqueness and convergence), with further concerns arising around the impact that such extensions have on the efficiency of the model.

\footnotetext{
${ }^{1}$ www.socasites.qub.ac.uk/mvanwalstijn/eusipco16/
}
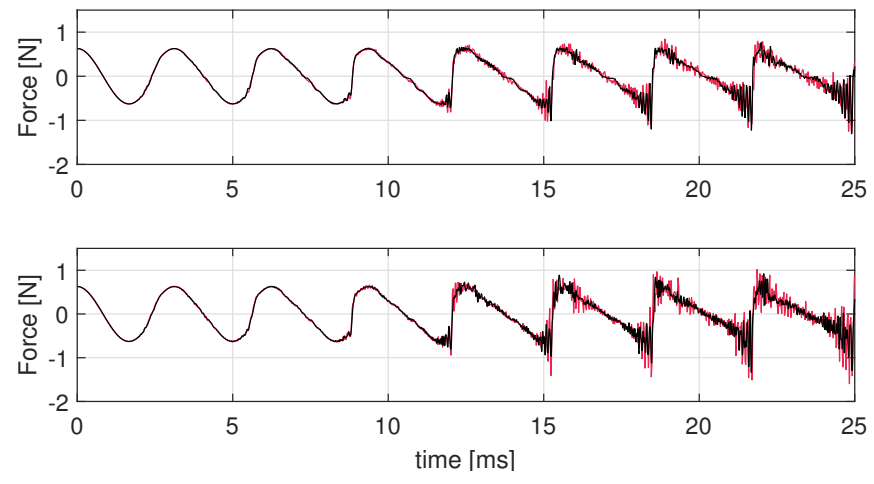

Fig. 4. String force at $x=L$, as simulated at $f_{\mathrm{s}}=44.1 \mathrm{kHz}$ (red line) and $f_{\mathrm{s}}=$ $705.6 \mathrm{kHz}$ (black line). Top: two-point scheme. Bottom: three-point scheme.

\section{REFERENCES}

[1] E. Rank and G. Kubin, "A waveguide model for slapbass synthesis," in IEEE Int. Conf. Acoust., Speech, \& Sig. Proc., vol. 1, 1997, pp. 443-446.

[2] A. Krishnaswamy and J. Smith, "Methods for simulating string collisions with rigid spatial obstacles," in Proc. IEEE WASPAA, New York, 2003.

[3] S. Siddiq, "A Physical Model of the Nonlinear Sitar String," Archives of Acoustics, vol. 37, no. 1, Jan. 2012.

[4] G. Evangelista and F. Eckerholm, "Player-instrument interaction models for digital waveguide synthesis of guitar: Touch and collisions," IEEE Trans. Audio, Speech, Lang. Proc., vol. 18, no. 4, pp. 822-832, 2010.

[5] D. Kartofelev, A. Stulov, H. Lehtonen, and V. Välimäki, "Modeling a vibrating string terminated against a bridge with arbitrary geometry," in Stockholm Musical Acoustics Conf., 2013.

[6] L. Trautmann and R. Rabenstein, "Multirate simulations of string vibrations including nonlinear fret-string interactions using the functional transformation method," Appl. Signal Proc., vol. 7, pp. 949-963, 2004.

[7] M. Rath, "Energy-stable modelling of contacting modal objects with piece-wise linear interaction force," in Proc. Int. Conf. Digital Audio Effects (DAFx-08), 2008.

[8] S. Bilbao, A. Torin, and V. Chatziioannou, "Numerical modeling of collisions in musical instruments," Acta Acustica u/w Acustica, vol. 101, no. 1, pp. 155-173, 2015.

[9] V. Chatziioannou and M. van Walstijn, "Energy conserving schemes for the simulation of musical instrument contact dynamics," J. Sound and Vibration, vol. 339, pp. 262-279, 2015.

[10] M. van Walstijn and V. Chatziioannou, "Numerical simulation of tanpura string vibrations," in Int. Symp. on Musical Acoustics (ISMA), 2014.

[11] S. Bilbao, "Numerical Modeling of String/Barrier Collisions," in Proc. of the Int. Symp. Musical Acoustics (ISMA), 2014.

[12] J. Woodhouse, "Plucked guitar transients: Comparison of measurements and synthesis," Acta Acustica u/w Acustica, vol. 90, no. 5, pp. 945-965, 2004.

[13] M. van Walstijn, J. Bridges, and S. Mehes, "A real-time synthesis oriented tanpura model," in Proc. Int. Conf. Digital Audio Effects (DAFx16), Accepted for Publication, 2016.

[14] S. Bilbao, Numerical Sound Synthesis. Wiley \& Sons, 2009.

[15] P. Deuflhard, Newton methods for nonlinear problems: affine invariance and adaptive algorithms. Springer, 2004.

[16] A. Meenakshi and C. Rajian, "On a product of positive semidefinite matrices," Linear Algebra and its Applications, vol. 295, no. 1-3, pp. 3-6, 1999.

[17] J. Ortega and W. Rheinboldt, Iterative Solution of Nonlinear Equations in Several Variables. SIAM, 1970, vol. 30.

[18] H. Cabannes and A. Haraux, "Mouvements presque-périodiques d'une corde vibrante en présence d'un obstacle fixe, rectiligne ou ponctuel," Int. J. Non-Linear Mech., vol. 16, pp. 449-458, 1981.

[19] C. Vyasarayani, S. Birkett, and J. McPhee, "Modeling the dynamics of a vibrating string with a finite distributed unilateral constraint: Application to the sitar," J. Acoust. Soc. Am., vol. 125, no. 6, pp. 3673-3682, 2009.

[20] S. Bilbao and A. Torin, "Numerical modeling and sound synthesis for articulated string/fretboard interactions," J. Audio Eng. Soc., vol. 63, pp. 336-347, 2015. 